\title{
Rousseur fatale dans La peau blanche de Joël Champetier
}

Fanie DEMEULE, Université du Québec à Montréal

Le présent article s'intéresse à la figure de la femme fatale rousse dans le roman d'horreur québécois La peau blanche (2003) de Joël Champetier. Si les criminelles de Champetier reprennent des traits stéréotypiques liés à la rousseur genrée au féminin, tels que la sexualité perverse et la criminalité, nous observerons comment ces personnages offrent aussi un potentiel féministe progressiste, entre autres parce qu'ils constituent un groupe féminin non repentant et victorieux. Dans un premier temps, je propose une lecture historique afin de faire ressortir les transformations de la figure de la femme fatale rousse dans l'imaginaire occidental. Face à ses mutations diachroniques, je remarque chez elle la persistance d'un caractère ambigu qui expliquerait sa dimension dangereuse et inquiétante, que je lie à la sémiotique du feu qui lui a été associée.

$\mathrm{Si}$, comme le remarque Sébastien Hubier dans Jeune et blonde rousseur (2015), la rousseur des femmes avait autrefois une connotation négative, elle se dote aujourd'hui d'une aura de pouvoir et d'agentivité. Cependant, Hubier remarque que toute inversion suppose une forme de continuité, laquelle est d'autant plus renforcée dans l'imaginaire postmoderne qui abandonne toute prétention aux idées de nouveauté et de progrès pour revisiter les formes du passé (29). C'est cette tension entre persistance et subversion de la figure de la rousse fatale que j'analyse chez Champetier.

\section{Rousseur et perversité}

Pour John Fiske, dans le cadre d'une étude d'objet de la culture populaire, aucun texte ne peut se comprendre sans une analyse préliminaire de son contexte intertextuel (1988). C'est pourquoi j'explore d'entrée de jeu les fondements de l'imaginaire de la femme fatale rousse, figure archétypale sexualisée dont la lascivité met en péril l'homme et la masculinité.

Ce qui distingue très tôt le mythe magdaléen, cette femme vêtue de ses seuls cheveux comme d'un vêtement impudique, réside dans ce rapport métonymique entre chevelure et corps féminin sexué. Comme le remarque Valérie André, « nombreuses sont les cultures à investir les cheveux des femmes d'un redoutable pouvoir érotique » (11). Ensuite, ce qui est particulier chez 
Marie-Madeleine est que celle-ci a toujours été imaginée et représentée rousse, car elle est devenue l'image même de la pécheresse (11). Au moment où elle s'est couverte de ses cheveux pour cacher sa nudité s'est cristallisée l'image de la rousseur féminine sexualisée dans l'imaginaire occidental. Cependant, la formation de cette image puise à des signifiants entourant la rousseur déjà en place depuis l'Antiquité, période lors de laquelle la pensée analogique domine. En effet, si Aristophane considère les blonds comme étant « dotés du courage du lion », il trouve les roux « fourbes comme le renard » (André 51). C'est d'ailleurs durant l'Antiquité que serait apparu le terme « roux », celui-ci empruntant son nom au feu, «pyrrhos », lui-même dérivé du prénom du fils d'Achille, le héros Pyrrhus (Hamilton 251).

Comme le souligne André, le lien de parenté entre la rousseur et les flammes, rédemptrices ou infernales, est bien ancré dans l'imaginaire collectif. Si le cheveu roux possède l'apparence flamboyante des flammes, il en partage de facto la symbolique ambivalente. Ainsi que l'expose Gaston Bachelard dans son essai sur la sémiotique du feu : «Le feu est un phénomène privilégié qui peut tout expliquer. [...] Parmi tous les phénomènes, il est vraiment le seul qui puisse recevoir aussi nettement les deux valorisations contraires; le bien et le mal. Il brille au Paradis, il brûle en Enfer » (André 18). Faisant écho à ces propos, Christiane Noireau soutient que « sans doute, le noir et le blond ont-ils souvent séduit. Mais la couleur qui flamboie, le roux et toutes ses nuances, a fasciné. Elle a aussi horrifié. Couleur parfaite portant en elle-même les conditions de possibilité de ses propres antinomies » (115).

Tenter de définir la relation entre la femme rousse et la perversité revient à poser la question de l'œuf ou la poule, tant leur rapport d'association se fonde sur un préjugé dont l'origine est difficile à cerner : les rousses souffrent de leur ressemblance avec Marie-Madeleine, MarieMadeleine est rousse parce que le roux est la marque de la différence. Il faut quand même reconnaître que la rareté naturelle de cette couleur lui confère d'emblée un caractère marginal. Selon l'ethnologue Mary Douglas, les êtres porteurs d'un signe physique divergent seraient éminemment craints et respectés par la communauté, étant à la fois considérés comme vulnérables et particulièrement puissants (99). Leur statut indéfinissable fait en sorte qu'ils sont considérés comme liminaux, ce qui les rend dangereux (99). Dès lors, ce qui fonde le pouvoir de la femme rousse est sa différence physique, perçue à la fois comme marginalité dangereuse et comme ambiguité vectrice de fabulation. 


\section{Rousseur et fatalité}

Devant ce portrait des origines de son imaginaire, on voit émerger le profil de la femme fatale rousse telle qu'il se manifestera à l'ère victorienne, à savoir comme icône de la fascination qu'exerce la sexualité féminine sur les hommes. Selon Hubier, au dix-neuvième siècle, la rousseur conserve son pouvoir de fascination au sens où elle suscite conjointement l'attirance et la défiance. Comme il l'observe, les artistes symbolistes et préraphaélites font de la rousseur une allégorie tout à la fois du péché, de la vitalité naturelle, de la marginalité et des destins contrariés (50).

Dans la littérature, l'image de la rousse perverse ou criminelle hante le roman de la fin du dix-neuvième siècle, où elle devient une source d'inspiration féconde pour les poètes et romanciers, qui exploiteront ses dimensions érotiques, énigmatiques et dangereuses. De même, selon les études considérées alors scientifiques de C. Lombroso et W. Ferrero, la rousseur affecterait davantage les «prostituées-nées» que les «femmes normales » (426). Ainsi, l'imaginaire scientifique de l'époque était au diapason avec la littérature. Si la sexualité féminine est animalisée et mortifère aux yeux des contemporains, celle de la femme rousse est d'autant plus dérangeante en raison de son caractère marginal, et la fatalité qu'elle représente est proportionnellement plus importante.

Maintenant que j'ai problématisé les liens entre la rousseur et sa dangerosité, définissons ce que j'entends par «femme fatale ». S'il est possible de retracer des femmes fatales des premières heures chez Lilith, ou Marie-Madeleine, ou encore chez les Amazones, ces figures féminines cathartiques, la figure de femme fatale n'est véritablement façonnée et nommée dans l'imaginaire occidental qu'à la période romantique de la seconde moitié du dix-neuvième siècle. D'après Béatrice Grandordy, ses caractéristiques les plus marquantes découlent de son absence de spiritualité et d'inhibition, car elle serait plutôt gouvernée par ses instincts (46). Ce faisant, elle s'apparente à une bête prédatrice, dangereuse pour l'espèce, car incapable de dominer l'animal en elle. Pourtant, malgré sa connotation péjorative ou repoussante, la fatale incarne aussi une sublimation, un enjolivement du féminin : «La femme fatale est l'expression des peurs et des angoisses, mais mâtinées d'une reconnaissance [...], d'une acceptation résignée, si ce n'est admirative. Elle impose un certain respect ou elle tient l'homme en respect » (47).

Je considère la femme fatale comme une métafigure qui recoupe plusieurs autres figures ou sous-figures, fantastiques ou non. Qu'elle soit vampire, sorcière, maîtresse ou prostituée, elle demeure ensorcelante, démoniaque et mortifère. Elle est cette féminité dangereuse, incarnant, 
selon André, « tous les fantasmes de l'Occident chrétien » (158). C'est une femme dont la seule présence dérange, puisqu'elle met en péril le pouvoir masculin. Si elle est menaçante sur le plan idéologique, cette figure l'est aussi sur le plan corporel, dans la mesure où le corps de la femme et sa sexualité sont chez elle associés à la mortalité. Lyndan Hart souligne que la femme énigmatique freudienne et la criminalité sont deux notions interdépendantes : « in the figure of the woman as criminal, the essence of femininity meets the alterity of the feminine. And they turn out to be the same thing » (36). La figure de la femme fatale est née de cette association. Comme le remarque Hart, même quand les femmes représentent le danger et la violence, elles demeurent paradoxalement incapables de supporter leurs actes agressifs. Nous avons donc, d'une part, des femmes innocentes, empathiques, douces, incapables de commettre un acte violent sans en subir les conséquences désastreuses. D’autre part, « a woman who is capable of aggression and violence becomes the masculine woman, the lesbian, the 'other' » (36). Cette association entre femme violente et altérité nous donne, entre autres, les archétypes sexualisés et inquiétants de la succube et de la sorcière. Si elle se rapproche de la sorcière dans sa représentation de la féminité surnaturelle et menaçante, la succube est une entité démoniaque qui utilise l'accouplement pour parasiter l'énergie vitale de ses proies masculines.

Malgré son portrait archétypal aux contours relativement balisés, la femme fatale demeure une figure qui « échappe à une définition incontournable, et lorsqu'elle l'incarne, elle peut ne le faire que partiellement» (Grandordy 9). Pour reprendre la définition de Grandordy, « c'est une femme ou un personnage usuellement féminin, dont le comportement conscient ou inconscient vise à amener l'homme à sa déchéance ou à sa perte ou à le placer dans une situation humiliante » (10). Malgré leurs grandes disparités, toujours selon Grandordy, « [le] commun dénominateur de ces créatures, de fiction ou de légendes historiques, réside dans le danger qu'elles présentent pour l'homme, le menant à sa perte, en usant avant tout, mais pas toujours, de séduction » (11). Ces femmes sont fatales pour l'homme, car elles mettent en lumière l'échec de la masculinité qu'il représente. Ainsi, les méfaits de la figure de la femme fatale existent avant tout dans la subjectivité d'autrui, et d'abord dans celle des hommes. En effet, comme le formule avec justesse Grandordy, « poser la question de la femme fatale, c'est poser, sociologiquement, celle de la misogynie, et littéralement celle du sens de l'adjectif fatal »(13).

Pour la femme fatale, le charme est à la fois une arme et un appât lui permettant de séduire afin de parvenir à ses fins. Le cinéma hollywoodien des premiers temps a particulièrement misé 
sur cette dynamique dans la conception de la figure filmique, directement inspirée de celle des artistes romantiques. Le genre du film noir, avec des œuvres telles que Sunrise (Murnau, 1927) ou Metropolis (Lang, 1927), met typiquement en scène un personnage féminin s'inscrivant en filiation directe avec la fatale décadentiste qui, en plus d'en récupérer le nom, déploie son capital sexuel similairement outrageux. Dans l'industrie naissante du cinéma, la fatale, souvent rousse, comme Gilda (Vidor, 1946), devient l'image médiatique d'une figure à consommer de manière scopophilique. Comme le relève Grandordy :

Cette association de la féminité à la sexualité traduit le rôle prédominant de la femme dans la culture visuelle du capitalisme : celui de l'incarnation du désir associé à la marchandise. Son potentiel de séduction est lié à l'idée que la femme est désirable, mais aussi destructrice à cause de sa sexualité. Le cinéma hollywoodien s'est approprié ce stéréotype dès ses débuts, et a fortement contribué à la médiatisation de cette figure de la séduction féminine. À travers ce stéréotype et son image, la femme, la femme fatale, s'associe à la figure naissante de la star, qui va être diffusée par l'industrie du spectacle alors en expansion. (188)

Cependant, la femme fatale du film noir possède un pouvoir d'agentivité faisant d'elle une femme ingouvernable, car non seulement on ne la manipule pas, mais de plus c'est elle qui obtient ce qu'elle veut: l'accomplissement du destin qu'elle s'est choisi (Grandordy 14). La poursuite entêtée de ses objectifs ne promet pas de concession ou de compromis, « elle veut qu'on lui donne ce qu'elle refuse de donner en échange » (14). La fatale est mystérieuse, fuyante, au point où, d'un œil externe, il est impossible de comprendre ses motivations profondes lorsqu'on tente de lire ses intentions. Comme le font remarquer Clara Serra Sanchez et Eduardo Fernandez Rubino :

[O]n a généralement tendance à désigner ce qui relève de la cachotterie, de la manigance, de la discrétion, de la clandestinité, en utilisant des termes « féminins ». Les femmes ont toujours été associées aux stratégies secrètes et aux complots de l'ombre. Comme les sorcières, les femmes pactisent dans le noir, et masquent leurs véritables intentions. [...] Elles sont menteuses, fausses, tentatrices. Cette représentation de la femme sert d'avertissement au monde masculin ; il faut se méfier des femmes, car elles ne sont pas ce qu'elles paraissent être. (cités dans Iglesias 248)

Alors que les objectifs du personnage masculin sont souvent explicités ou illustrés de manière claire, ceux de la fatale sont typiquement impénétrables ; en ce sens, sa figure récupère le trope du féminin insondable, car trop complexe, éternellement énigmatique. 


\section{Fatales contemporaines}

Qu'en est-il dans les figurations contemporaines de la rousseur fatale ? Dans les productions de genres, elles possèdent toujours cette aura d'unicité qui les distingue des autres femmes et les maintient dans une certaine mesure en marge de l'humanité ; elles demeurent les femmes marginales, perturbatrices de l'ordre social. On assiste encore aujourd'hui à un certain maintien du rapport corrélatif entre rousseur et sexualité, mais il s'agit maintenant davantage de la femme rousse et de $s a$ sexualité. On peut penser, entre autres, à Jolene (Jolene, Dan Ireland, 2008) ou à Maude Lebowski (The Big Lebowski, Frères Coen, 1998). En effet, son arc narratif correspond à celui d'une ascension au pouvoir individuel à travers une réappropriation de son corps dont le capital érotique et sexuel lui revient de tout droit ; une autonomisation qui ne lui coûte plus nécessairement la vie - sauf exception (Thelma and Louise, Ridley Scott, 1991).

À travers leur reprise insistante de plusieurs des traits caractériels des figures rousses, on retrouve chez ces personnages contemporains une dimension métadiscursive réfléchissant les paramètres mêmes de leur rousseur fatale. Selon Hubier, encore aujourd'hui : « les figurations de la rousseur semblent renvoyer univoquement à une féminité sulfureuse, transgressive, incendiaire - criminelle, peut-être. Et, tout naturellement dans une société hypermoderne fascinée par les femmes déterminées, la rousseur est devenue tendance, dans l'intensité d'une lumière plus que d'une couleur» (21). On peut noter une forme de subversion lorsque la narration installe la possibilité que la femme fatale masque consciemment ses desseins, et que l'on découvre en tant que lecteur ou spectateur que cette occultation participait activement à la bonne marche de son plan d'action. Les dévoilements de ces manigances nous permettent de « comprendre ces stratégies comme des moyens légitimes de lutte pour la survie au sein de l'espace limité et réduit auquel ils [les personnages de femmes fatales] sont cantonnés » (Sanchez et Rubino, cités dans Iglesias 248). Ce faisant, le personnage de la femme fatale tord non seulement le cou au stéréotype de femme victime de son propre charme, mais manipule également l'horizon d'attente du public en déjouant ses anticipations. De cette façon, les auteurs confèrent un certain pouvoir à un personnage de femme fatale à même de triompher grâce à son pouvoir personnel, qui n'est donc plus victime de ce pouvoir. Ce renversement de la fatalité liée au pouvoir permet ainsi de repenser les rapports entre femme et agentivité. 


\section{Une peau abjecte}

Certaines œuvres littéraires contemporaines offrent des portraits de femmes fatales rousses qui reprennent certains motifs traditionnels tout en les revisitant de l'intérieur. C'est le cas des criminelles rousses du roman La peau blanche, paru en 1997 aux éditions Alire, de l'auteur québécois Champetier, qui font écho aux figures de rousses fatales antérieures. En 2004, l'œuvre est adaptée au cinéma par Daniel Roby et est scénarisée par Champetier lui-même. Notons à l'égard de l'affiche du film la position de la jeune femme, nettement plus dominante que son partenaire masculin dans la composition de l'image. En plus du regard perçant qu'elle jette au spectateur, elle est d'emblée placée en position de pouvoir. Le titre La peau blanche fait d'ailleurs référence au teint de ce personnage, Claire Lefrançois, ainsi qu'à celui de ses consœurs succubes, des rousses « à la peau presque transparente » (Champetier 73).

C'est précisément cette peau blême qui dégoûte le narrateur, Thierry Guillaumat, qui d'ordinaire a horreur des roux et rousses : «Ce n'est pas une question de couleur de cheveux. C'est la peau. Cette peau trop blanche, trop mince, trop fragile, cette peau à travers laquelle les veines transparaissent, qui donne l'impression de voir la chair qui est en dessous. C'est cette intimité forcée qui m'est insupportable, qui me soulève le cœur» (Champetier 73). On comprend que ce qui répugne Thierry par-dessus tout est l'aspect organique de l'existence humaine, que lui révèle la transparence de la peau blanche. Or, malgré le fait qu'elle soit une rousse au teint très clair, Thierry est subjugué par Claire Lefrançois - ce qui offre dès leur rencontre un indice de la nature surnaturelle de la jeune femme, une succube. Sur un ton apocalyptique, le résumé de quatrième de couverture insiste sur l'invasion grandissante de ces succubes et de leur aspect fatal : «Elles sont parmi nous... Et elles sont mortellement dangereuses ! »

Dans toutes les mythologies, séduction, dévoration et mort sont des thèmes communs de tous les êtres démoniaques femelles et ils sont conjointement revisités par Champetier à travers la sororité des Lefrançois. Se nourrissant de sperme, de sang et de chair d'hommes (noirs de préférence), ces femmes blanches sont posées en polarité extrême avec les hommes de race noire, leur nourriture de prédilection. Henri, l'ami haïtien du narrateur, avance le fait que les Noirs seraient les premiers êtres humains, comparativement aux succubes rousses, qui elles, seraient une mutation récente du génome (104). La jeunesse de leur race est la raison pour laquelle ces femmes doivent impérativement se nourrir des humains de souche première afin de survivre. 
À l'image du texte lui-même, à cheval entre le thriller, le roman noir, le policier, le romance novel, le buddy novel, la comédie, l'horreur et le drame psychologique, la figure de la succube est elle aussi hybridée, laissant entrevoir d'autres mythes de femmes fatales, soit la sirène et la Gorgone. Par exemple, la scène de rencontre entre Thierry et Claire évoque une plongée dans le gouffre abyssal d'une sirène chantante, se soldant en une «médusation » préfigurant l'issue finale tragique du narrateur :

à travers le bourdonnement sourd qui habitait l'université comme une lente respiration, des notes cristallines flottaient jusqu'à mon balcon, pareilles à des bulles sonores. [...] J'ai longé les couloirs anonymes jusqu'à un escalier et je suis descendu jusqu'au niveau du métro. [...] Mais ce n’était pas la musique, aussi exquise fût-elle, qui avait raidi ainsi tous mes membres. C'était la claveciniste. Combien de temps suis-je resté là, adossé contre un panneau d'affichage, immobile, pour ne pas dire statufié ? La beauté de la claveciniste outrepassait toute réalité physique, c'était un être de lumière, une flamme qui me laisserait pour l'éternité une cicatrice dans le cour et l'esprit. (48)

Avec ses nombreuses références allant des mythes aux récits bibliques en passant par Michel Tremblay et David Cronenberg, le texte de Champetier s'inscrit dans une intertextualité réflexive qui en fait un bricolage postmoderne conscient de ses effets. D'ailleurs, le personnage d'Henri compare d'entrée de jeu Claire à une femme fatale de film noir lorsqu'il dit à Thierry : "Superbe rousse. Adresse inconnue. Baise comme une salope. Tu sais, ça fait très film noir » (57). Selon l'auteur lui-même : «Dans La peau blanche, on reprend les clichés et les stéréotypes pour les revisiter de l'intérieur »(DVD, commentaires audio). Pour ces raisons, le récit de Champetier s'inscrit dans un réinvestissement postmoderne de la rousseur fatale et de sa stéréotypie, dont il en est manifestement conscient et avec lequel il joue avec les codes. En tant qu'auteur de littérature de l'imaginaire, Champetier s'adresse à un lectorat sans doute lui aussi familier avec les genres de l'imaginaire, et c'est à partir de ce rapport de connivence que s'établit le jeu de tension entre répétitions et écarts envers les normes génériques.

\section{Décadentes et victorieuses}

Marquise, la plus jeune des trois sœurs, reprend l'image ultime de la rousseur décadente ; prostituée, criminelle, violente de parole et de geste. Quant à elle, Claire, comme son nom l'indique, offre des abords plus innocents, voire vertueux ; elle reste sage et discrète, porte des vêtements pudiques, ne boit pas et ne fume pas. Elle se montre très posée, sauf au lit avec Thierry, où elle se transforme, sans surprise, en amante torride. Cependant, sous ses allures ingénues se 
cache une véritable renarde, car c'est bien celle des trois sœurs qui parvient à obtenir une source de nourriture à long terme en amadouant profondément Thierry. En effet, Claire gagne le cœur et la confiance de celui-ci en jouant la carte de l'amoureuse, en prétendant vouloir le protéger d'ellemême : « - Je ne t'ai jamais voulu de mal. Jamais. [...] C'est pour ça qu'il faut que je te quitte » (188). Elle sait très bien que ces paroles, loin de le dissuader, auront pour effet de redoubler ses ardeurs. Or, comme se plaît à le rappeler Hubier, dans l'imaginaire, il n'est point de rousse qui ne soit, d'abord et avant tout, une actrice (46). Grâce à une narration à focalisation interne agilement assurée par le point de vue (limité) de Thierry, il faut se rappeler que les desseins des rousses de Champetier sont non seulement cachés aux yeux du protagoniste, mais aussi aux yeux du lecteur.

À propos de ce point de vue masculin, nous ne pouvons certainement nier la dimension scopophilique d'une telle monstration de femmes littéralement «croqueuses d'hommes » comme union fantasmatique masculiniste d'Éros et de Thanatos puisant dans un sempiternel male gaze, qui se traduit chez Champetier dans des scènes nouant jouissance sexuelle et dévoration. La description du cadavre d'Henri se faisant déguster par Marquise en est assez révélatrice :

Marquise m'a regardée, le regard brumeux, la bouche entrouverte sur un demisourire. Entre ses lèvres luisantes de sang. [...] D'une main elle recueillait dans le fond de la baignoire le sang qui avait coulé du cadavre éventré d'Henri, le cadavre sur lequel elle était assise. Elle portait ensuite la main à sa bouche qu'elle léchait avec la préciosité et la gourmandise d'une chatte. De son autre main ensanglantée, elle se masturbait profondément. Elle gémissait sous une double extase ; ses cheveux gluants de sang frémissaient parfois sous un spasme et maculaient le carrelage de gouttes écarlates. (Champetier 225)

Cette sexualité s'avérait mortifère dans les représentations antérieures, qui se soldaient bien souvent par la «punition » de la femme fautive, soit sous la forme d'un châtiment physique ou symbolique, ou encore par sa mort.

Au contraire des anciens récits qui les sacrifiaient, le récit de Champetier dépeint la victoire des femmes fatales. Désormais, elles ne semblent plus être victimes de la fatalité qui les accompagne, mais s'en servent comme d'un instrument de survie, tout en rejetant l'étiquette même de monstre, ainsi qu'en témoigne la mère Lefrançois à ce sujet : «Vampires. Ceux qui ne croient pas en notre existence pourraient nous appeler comme ça. [...] Au fond, nous nous préoccupons peu de savoir ce que nous sommes. Nous existons. Ça nous suffit. Nous avons un rôle à jouer et nous le jouons, c'est tout » (231). La rousseur fatale ne se révèle plus être l'apanage d'un démon surnaturel, mais est plutôt liée à une mutation génétique naturelle de la race humaine. De même, 
les justifications survivalistes du cannibalisme problématisent la monstruosité dont on pourrait étiqueter ces femmes, et par cet ébranlement, les questions spécistes et posthumanistes soulevées mettent en doute la valeur de l'être humain. Vers la fin du roman, la mère Diane Lefrançois questionne le narrateur à ce propos : « Thierry, je te le demande: être un humain, est-ce si glorieux ? Qu'est-ce que c'est, un humain ? Qu'est-ce qu'ils ont fait de bon, ces humains, qu'ils soient noirs, blancs, jaunes, rouges ou bleus ? » (232). À travers ce prisme nous entrevoyons l'ébranlement de l'idée de cruauté, ainsi qu'un effondrement des polarités morales à travers un rapprochement des violences communes nécessaires à la survie des espèces : «Au fond, nous ne sommes pas différentes de n'importe quelle créature vivante, Thierry. Tout ce que nous voulons vraiment, c'est survivre » (233). Selon Étienne Balibar, la cruauté est cette part « dépourvue de sens » et «non justifiable » de la violence (71). Qu'est-ce que la violence alimentaire de ces êtres comparativement à la violence interraciale organisée entre les humains, mais aussi celle des humains envers les autres espèces du vivant? Par l'entremise de cette rousseur fatale aux accents finalement plus eschatologiques que fantastiques, le récit de Champetier interroge ce qui compose l'humanité et ses valeurs, sa violence comme sa cruauté.

L'amour rend aveugle, dit-on. C'est particulièrement vrai chez le narrateur Thierry, si médusé par sa belle rousse qu'il en oublie la violence grandissante que celle-ci perpétue, dont il est d'abord témoin, puis victime. En résulte une narration haletante qui témoigne du développement de l'obsession du narrateur envers Claire Lefrançois : « elle habitait maintenant mes pensées jour et nuit, m'empêchait d'étudier, de dormir, de lire. Claire, Claire, Claire...Tout, tout le temps, me ramenait à elle » (Champetier 97). À un certain point, même si Thierry prend connaissance de l'ampleur de son obsession provoquant sa propre déchéance, il n'est plus en mesure de se défaire de l'envoûtement de Claire - il ne l'a jamais été d'ailleurs. Littéralement vampirisé par son amoureuse, Thierry s'affaiblit, ce qui se traduit dans sa narration de plus en plus désaffectée et sporadique. Il finit par se soumettre entièrement à la race matrilinéaire des rousses, abdication qui signe la fin du roman. Ironiquement, alors qu'il était d'abord révulsé par la corporéité humaine que lui rappelait la peau blanche, Thierry se transforme lui-même en un simple morceau de viande. En épilogue, le narrateur parle de sa mort prochaine : détaché, il se demande pragmatiquement qui nourrira sa fille une fois qu'il disparaîtra définitivement, dévoré par cette dernière, sa femme et ses belles-sœurs. Ainsi, on comprend que, d'un repas à l'autre, les femmes rousses monteront au pouvoir. 


\section{Communauté de fatales}

À la différence des récits antérieurs tendant à isoler les femmes fatales, le récit de La peau blanche les rassemble. À la manière de néo-amazones, ces succubes rousses vivent entre elles, en retrait de la société. Dans le contexte de la société hautement patriarcale qui l'a vu naître, le mythe des Amazones est une projection aux antipodes du féminin normatif de la Grèce antique, l'image même de la marge et de son danger. Pour Cécile Voisset-Veysseyre dans Des Amazones et des femmes, elles incarnent l'extrême féminin hérétique et insoumis, et leur caractère barbare souligne de manière moralisatrice la menace que représentent les femmes s'écartant des normes prescrites ; elles deviennent, ni plus ni moins, ennemies du peuple et, de fait, illustrent le désordre encouru en l'absence du patriarcat (7).

Récemment, Coline Cardi et Geneviève Pruvost constataient une récurrence des fins tragiques pour les récits impliquant des Amazones : «On se contentera de constater que les fictions apaisées, enchantées et utopiques, présentant comme allant de soi des Amazones sous la forme d'une happy end, sont rares » (47). Or, je constate aujourd'hui le phénomène inverse, soit le triomphe final de ces femmes que je qualifie de Néo-Amazones. C'est notamment ce qui se produit chez Champetier, tandis qu'on assiste à la montée en puissance finale du matriarcat des succubes.

Derrière ce matriarcat se dresse l'importance du lieu de rassemblement, qui est à la fois un point de repère et un refuge pour ses membres. Dans le roman de Champetier, il s'agit de la maison familiale longueuilloise, où les femmes de la famille Lefrançois vivent en retrait. Lorraine Code explique ce lieu refuge de manière éclairante ; pour elle, lorsque les femmes se soutiennent mutuellement, elles sont à même de créer leur propre espace d'autorité. Selon Code, la création d'un tel espace dépend d'une volonté et d'un effort collectif de femmes en mesure de se prêter confiance entre elles (243). Représenter de tels espaces de pouvoir dans l'imaginaire permet, selon Sharon Ross, d'illustrer la viabilité de sites de communications et d'interactions non orthodoxes (233). Code considère que la communication doit avant tout prendre racine dans un lien communautaire sororal (238). Si le féminisme porte la différence sexuelle à travers une critique du système genré dominant comme un pouvoir institutionnel (Braidotti 215), la sororité envisage la résistance face à l'instrumentalisme de tout acabit. Pour Marcela Lagarde, dans « Intimité et sororité », la filiation sororale entend l'ingouvernabilité des femmes entre elles, un prendre soin organisationnel indépendant du système central. Bien que ce système se dote de teintes violentes

et horrifiques chez Champetier, il n'en reste pas moins que le roman représente un groupe 
autonome de femmes s'entraidant et progressant ensemble. Par ailleurs, Sandra Sheehy soutient l'importance des représentations de groupes de femmes et leur influence sur la manière dont les femmes réelles considèrent de tels liens.

\section{Conclusion}

En raison des questions morales qu'il soulève, le roman de Champetier peut difficilement nous laisser indifférents. Que penser de la violence des néo-fatales rousses chez Champetier, notamment celle, explicite, de la cadette Marquise ? Et surtout, quel est le potentiel féministe de ce genre de représentation faisant perpétrer la violence sur une figure féminine aux traits humanisés?

À partir d'une étude de la violence au féminin dans un corpus littéraire québécois, Paula Ruth Gilbert constate que la manière dont la société parle et représente les femmes et leur usage de violence a un impact sur la façon dont les femmes sont perçues et traitées dans le système judiciaire réel (1271). En se basant sur Foucault, Gilbert explique que, si on peut revendiquer un discours pluriel sur la sexualité (comme c'est le cas dans Surveiller et punir), une telle multiplicité discursive est également nécessaire en ce qui a trait aux discours sur la violence, et spécifiquement dans le cas de nos perceptions du genre et des femmes. Selon Anette Ballinger, Laura Grindstaff et Martha McCaughey, la société a besoin de voir les femmes violentes criminelles pour comprendre que les femmes peuvent elles aussi être violentes, et être ainsi en mesure d'appréhender cette violence dans le réel. Quant à elle/lui, Judith/Jack Halberstam fait référence à June Jordan et à son concept de «lieu de rage ». Pour Halberstam, la représentation de violence impunie perpétrée par des femmes non repentantes envers des hommes ouvre un espace au potentiel cathartique propice à l'investissement émotionnel des lectrices et spectatrices (247). Selon Halberstam cette monstration de violence aurait le potentiel de désintégrer la « norme mythique » entendant les femmes comme douces et dociles, créant ainsi un espace de rage permettant de repenser les positions de pouvoir. En cela, l'espace de rage est un territoire pour la résistance (251). Toujours selon Halberstam, la représentation de la violence et de la rage des femmes est une condition sine qua non d'un changement social, la "violence imaginée » permettant selon elle «d'énerver le système » (94). Les lectrices et spectatrices qui accèdent à l'imaginaire du « œil pour œil, dent pour dent » peuvent se sentir investies du droit de riposte ; quant aux agresseurs potentiels, ils peuvent en venir à anticiper avec crainte une violence féminine 
adverse (8). « Nous devons être capables d'imaginer la violence - et notre violence doit pouvoir être imaginable - car le pouvoir du fantasme ne consiste pas à représenter le réel mais à le déstabiliser » (8). Tiina Vares montre une distinction de la part des spectatrices entre la « violence imaginée » (acceptable) et la « violence réelle non nécessaire » (inacceptable) (230). Elle observe cette dichotomie au moment où se rencontrent les contradictions apparentes dans le fait de cultiver une philosophie non violente tout en appréciant le spectacle de femmes violentes refusant la position de victime (230). Pour Vares, il est clair que dans un contexte social où les femmes sont souvent considérées comme des victimes, la violence des femmes dans la fiction est une donne invariablement politique (226). Selon elle, la violence des femmes fictives est une réponse symbolique à la violence systémique dont elles sont constamment les cibles (234).

Les parcours des succubes rousses de Champetier nous montrent un scénario où les femmes triomphent et gagnent en agentivité dans la violence. À cet égard, dans son essai The Force of Fantasy, Judith Butler suggère de repenser la relation entre réel et imaginaire en ne concédant plus au réel un a priori de stabilité (252). Pour Butler, le réel est une construction labile qui ne peut se percevoir qu'à partir d'un point de vue subjectif, qui lui, baigne dans l'irréel et le fantasmatique (252). Ce que nous percevons dans la violence imaginée est donc modelé par nos propres peurs comme nos désirs face à la violence réelle, et par-dessus tout par notre volonté de donner un sens au discours qui nous est proposé par la fiction.

Certes, il faut souligner le fait que la violence des rousses meurtrières dans La peau blanche demeure justifiée par la nécessité de s'alimenter, en plus d'être encore une fois sexualisée. Néanmoins, elle fournit une précieuse et trop rare représentation de communauté de femmes violentes, victorieuses et, surtout, non repentantes.

\section{Bibliographie}

André, Valérie. Réflexions sur la question rousse : Histoire littéraire d'un préjugé. Paris: Tallandier, 2007.

Bachelard, Gaston. La psychanalyse du feu. Paris : Gallimard, 1985.

Ballinger, Anette. Gender, Truth and State Power : Capitalising on Punishment. Londres : Routledge, 2016.

Braidotti, Rosi. Nomadic Subjetcs. Embodiement and Sexual Difference in Contemporary Feminist Theory. New York : Columbia UP, 2011. 
Cardi, Coline et Geneviève Pruvost. Penser la violence des femmes. Paris : La Découverte, 2017. Champetier, Joël. La peau blanche. Montréal : Alire, 1997.

Code, Lorraine. Rhetorical Spaces : Essays on Gendered Locations. New York: Routledge, 1995. Douglas, Mary. Purity and Danger. Londres: Routledge, 1966.

Grandordy, Béatrice. La femme fatale. Ses origines et sa parentèle dans la modernité. Paris: Harmattan, 2013.

Grindstaff, Laura et Martha McCaughey. Real Knockouts : The Physical Feminism of Women's Self-Defense. New York: New York UP, 1997.

Halberstam, Judith. «Imagined Violence/Queer Violence: Representation, Rage, and Resistance ». Social Text 37, A Special Section Edited by Anne McClintock Explores the Sex Trade (1993).

Hamilton, Edith. La mythologie : ses dieux, ses héros, ses légendes. Paris : Marabout, 2013.

Hart, Lyndan. Between the Body and the Flesh : Performing Sadomasochism. Philadelphie: Columbia UP, 1998.

Hubier, Sébastien. Jeune et blonde rousseur. Paris : Murmure, 2015.

Iglesias, Pablo. Les leçons politiques de Game of Thrones. Paris : Post-éditions, 2015.

Gilbert, Paula Ruth. Violence and the Female Imagination : Quebec's Women Writers Re-frame Gender in North American Cultures. Montréal : McGill-Queen’s UP, 2006.

--- et Kimberly K. Eby. Violence and Gender : An Interdisciplinary Reader. Montréal : Pearson, 2003.

Lagarde, Marcela. «Preface. Feminist Keys for Understanding Feminicide : Theoretical, Political, and Legal Construction ». Terrorizing Women. Feminicide in the Americas. Dir. RosaLinda Fregoso et Cynthia Bejarano. Durham : Duke UP, 2010. 8-21.

Lombroso, C. et W. Ferrero. The female offender. New York : Appleton, 1893.

Noireau, Christiane. L'esprit des Cheveux : Chevelures, poils et barbes, Mythes et croyances. Anjou : Cheminements, 2009.

Pastoureau, Michel. Rouge : Histoire d'une couleur. Paris : Seuil, 2016.

---, « Tous les gauchers sont roux ». La trahison. Le genre humain. Paris : Seuil, 1988. En ligne.

Sheehy, Sandra. Connecting : The Enduring Power of Female Friendship. New York : Morrow, 2000 .

Voisset-Veysserie, Cécile. Les Amazones font la guerre. Paris : Harmattan, 2010. 